\title{
Incoherent Excitation of Thermally Equilibrated Open Quantum Systems
}

\author{
Leonardo A. Pachón ${ }^{1,2}$ and Paul Brumer ${ }^{2}$ \\ ${ }^{1}$ Instituto de Física, Universidad de Antioquia, AA 1226 Medellín, Colombia \\ ${ }^{2}$ Chemical Physics Theory Group, Department of Chemistry and Center for Quantum Information and Quantum Control, \\ University of Toronto, Toronto, Canada M5S $3 H 6$
}

(Dated: August 17, 2018)

\begin{abstract}
Under natural conditions, excitation of biological molecules, which display non-unitary open system dynamics, occurs via incoherent processes such as temperature changes or irradiation by sunlight or moonlight. The dynamics of such processes is explored analytically in a non-Markovian generic model. Specifically, a system $\mathrm{S}$ in equilibrium with a thermal bath TB is subjected to an external incoherent perturbation BB (such as sunlight) or another thermal bath $\mathrm{TB}^{\prime}$, which induces time evolution in $(\mathrm{S}+\mathrm{TB})$. Particular focus is on $(i)$ the extent to which the resultant dynamics is coherent, and (ii) the role of "stationary coherences", established in the (S+TB) equilibration, in response to the second incoherent perturbation. Results for systems with parameters analogous to those in light harvesting molecules in photosynthesis show that the resultant dynamical behavior is incoherent beyond a very short response to the turn-on of the perturbation.
\end{abstract}

PACS numbers: 03.65.Yz, 05.70.Ln, 37.10.Jk

\section{INTRODUCTION}

The dynamics of open quantum systems (e.g., molecules of interest in contact with an environment) in the presence of an external perturbation is of great current interest and applicability. Two general circumstances can be envisioned, one where the external perturbation is "designed", the second where it occurs naturally. Within the framework of electromagnetic perturbations, popular examples of the former include pulsed laser studies of molecular dynamics in the laboratory [1, 2], laser based scenarios for quantum control and quantum information, various spectroscopies, etc. These artificial light sources are often characterized by pulses of short temporal duration and significant coherence times.

By contrast, natural light induced processes such as vision or photosynthesis are induced by virtually stationary chaotic blackbody light sources such as the sun, which have significantly different properties [3-8]. Similarly, natural processes, like ion transport through membranes [9] may be induced by temperature changes. Both perturbations, temperature change and excitation by natural light, are fully incoherent. That is, they are both associated, as outlined later below, with a perturbation described by a density matrix that is a mixture of stationary states.

A proper treatment of the systems described above requires that the system $\mathrm{S}$ of interest be first equilibrated with a background thermal bath TB, after which it is subjected to a second perturbation comprising a second thermal bath (denoted $\mathrm{TB}^{\prime}$ ) or blackbody radiation (denoted BB). Our focus here, in this two step process, is on the role of coherences in the dynamics.

One motivation for this work lies in recent studies of coherent quantum dynamics in model photosynthetic light harvesting systems $[1,10]$ and in vision $[2,4,5]$. Coherent dynamics of this kind is observed in experiments in which the system is excited with coherent laser light, and the timescales for the decay of the coherence, gen- erated by the system interacting with its environment, is then measured. Principal among the observations on molecules involved in photosynthesis is that the coherent dynamics associated with electronic energy transfer persists, at room temperature, on considerably longer time scales (e.g., 400 to $2000 \mathrm{fs}$ ) than is expected from earlier results on other systems [11-14], and from theories of decoherence [15]. A number of computational results have been obtained, and theories advanced, as to why such longevity occurs. For example, we have identified a number of physical conditions under which such long lived electronic coherence persists [16, 17].

The relevance of these results for realistic photosynthetic systems, or for the operation of devices that mimic photosynthesis, however, depends heavily on the relationship between the dynamics observed in the experiments, which use pulsed coherent light, and dynamics under natural light, such as that from the sun. Both recent [7] as well as earlier studies [3] on this relationship for isolated molecules (i.e. molecules that are not in contact with an environment) show that whereas pulsed coherent light induces dynamics in the molecule, natural stationary chaotic light does not. Rather, irradiating an isolated molecule with such natural light over long natural time periods creates a stationary mixture of molecular eigenstates.

Natural light absorbing molecules of interest in, e.g., photosynthesis and vision are not, however, isolated. Rather they are in contact with an external environment through which decoherence and relaxation occur. Hence, there is a need to understand the response of a system+bath that is subject to an incoherent excitation. A study of Retinal excitation [5] under these conditions confirmed that stationary eigenstates resulted in this open system as well. An alternate study on photosynthetic systems [6] simultaneously in contact in contact with both $(\mathrm{TB}+\mathrm{BB})$ noted the appearance of off-diagonal elements of the subsystem density matrix $\mathrm{S}$ and regarded them as "stationary coherences", a concept elucidated further 
below. The relation of these stationary coherences to dynamics was, however, unclear. Hence, clarifying the situation regarding open systems under incoherent perturbation is, therefore, well motivated.

As noted above and as emphasized, in this work it is important to recognize that the natural processes noted above occur in two distinct steps. That is, in the first step the subsystem $\mathrm{S}$ thermally equilibrates with its surrounding bath, yielding a result that we denote as $(\mathrm{S}+\mathrm{TB})$. In the second step this equilibrated system is placed in contact with a source of incoherent light (BB) or another thermal bath $\mathrm{TB}^{\prime}$ with which the system interacts, giving an $(\mathrm{S}+\mathrm{TB})+\mathrm{BB}\left[\right.$ or $\left.(\mathrm{S}+\mathrm{TB})+\mathrm{TB}^{\prime}\right]$ system. As described in detail below in Sec. II, the first generates "stationary coherences". The nature of the subsequent relaxation dynamics and the role of the stationary coherences arising from the equilibration of $\mathrm{S}$ with $\mathrm{TB}$ in the subsequent dynamics of $(\mathrm{S}+\mathrm{TB})+\mathrm{BB}$ [or of $\left.(\mathrm{S}+\mathrm{TB})+\mathrm{TB}^{\prime}\right]$ are described below.

The existence of substantial stationary coherences requires strong system-environment coupling (whether dissipative or not) $[18,19]$, and as we will show, are largest in the low temperature regime. This regime of strong coupling and low temperature is relevant in the context of, e.g., electronic energy transfer $[16,17]$. Being in this regime prevents the application of simplifying approximations to the evolution of the density matrix, such as the Markovian approximation [20] or secular approximation. That is why a thorough analysis of these off-diagonal terms and their influence on the dynamics have been elusive. Here we show, using an analytic model that these off-diagonal terms can contribute to the dynamics of the populations only when they enter as initial correlations between the system and the thermal bath, and that in realistic systems these contributions are small.

Note for clarity below, the specific characteristics of the natural process that are of interest. That is, we are focusing on molecular systems that are, as in natural photosynthetic processes, irradiated by blackbody radiation for time scales that are far far longer (e.g. hours) than the inverse of the molecular energy level spacing, which would define the time scale for coherent molecular dynamics. Results of this study are found to extend the result previously obtained for the isolated system to the open system. That is, "natural incoherent chaotic light" is shown to be incapable of inducing coherent dynamics in either isolated or open systems.

Note that the dynamics examined here takes place on a single electronic surface. The extension of this analytic influence functional approach to non-adiabatic processes is in progress. However, the general conclusions obtained here are expected to hold in the case of excitation from one electronic surface to another.

The paper is organized as follows: Sec. II introduces features of the first thermalization step, i.e. the process of $\mathrm{S}+\mathrm{TB}$ relaxing, with a focus on the concept of "stationary coherences". Section III provides the Hamiltonian under consideration and associated computational results for the
$(\mathrm{S}+\mathrm{TB})$ relaxation. The case of $(\mathrm{S}+\mathrm{TB})$ interacting with a second thermal bath is contained in Sec. IV, whereas the case where the perturbation is incoherent light is discussed in Sec. V. A short discussion and summary is provided in Sec. VI.

\section{EQUILIBRATION OF (S+TB) AND "STATIONARY COHERENCES"}

The essence of open quantum systems lies in the interaction between a part of interest, "the system", with a component which is not of interest "the environment" or "bath". Consider then the two components, a system $\hat{H}_{\mathrm{S}}$ with eigenbasis $\left\{n_{i}\right\}$ defined on the Hilbert space $\mathcal{H}_{\mathrm{S}}$ and (in this section) the thermal bath described by the Hamiltonian $\hat{H}_{\mathrm{TB}}$ with eigenbasis $\left\{N_{i}\right\}$ defined on the Hilbert space $\mathcal{H}_{\mathrm{TB}}$. In the absence of an interaction between them, the total Hilbert space $\mathcal{H}=\mathcal{H}_{\mathrm{S}} \otimes \mathcal{H}_{\mathrm{TB}}$ will be diagonal in the basis $\left\{n_{i} \otimes N_{i}\right\}$. However, if the systems interact via a coupling term $\hat{H}_{\mathrm{ST}}$, then $\mathcal{H}$ is no longer diagonal in this basis. To obtain information on the system, we would then trace over the degrees of freedom of the bath. It is then clear that the remaining sector $\mathcal{H}_{\mathrm{S}}$ is no longer diagonal in the $\left\{n_{i}\right\}$ basis. Rather, the new effective eigenbasis should "know about the thermal bath"; therefore the resultant effective system basis should be a function of the coupling to the bath and of the temperature.

In general, one is not interested in studying the dynamics in terms of this coupling-and-temperature dependent basis, but rather in describing the dynamics in the system eigenbasis of $\hat{H}_{\mathrm{S}}$. Viewed in this basis, the coupling to a bath generates off-diagonal elements in the system density matrix. Once the (system+bath) have relaxed to equilibrium these off-diagonal elements do not change with time, and are not associated with dynamics. Rather, they can be termed "stationary coherences". They are just a manifestation of our focus on the system rather than on the coupled system+bath.

An alternate perspective on these stationary coherences is that strong coupling to the environment causes overlap of homogeneous line shapes associated with different energy states of system $\mathrm{S}$.

Given that the equilibrated $\mathrm{S}+\mathrm{TB}$ composite is the natural state in which one finds the system $\mathrm{S}$ in the cases of interest, the relevant questions here are then: $(i)$ what are the nature and times scales of the dynamics when this equilibrated (system+bath) is exposed to an incoherent perturbation, and $(i)$ what is the role of the stationary coherences in this subsequent dynamics? For example, it has been suggested [6], but not explored quantitatively, that such terms are capable of inducing coherent dynamics when the equilibrated $\mathrm{S}+\mathrm{TB}$ is subject to an additional perturbation.

Below we show how to properly address the incoherent excitation of a system $\mathrm{S}$ in a thermal environment by, in contrast with previous work [4-6], considering the exci- 
tation in two steps. In particular, using an analytically soluble model valid in the whole range of parameters, we study the role of environment-generated coherences during: $(i)$ thermalization of an initially isolated central system $\mathrm{S}$ in contact with a thermal bath TB, and (ii) the subsequent dynamics induced by the presence of a second thermal bath $\mathrm{TB}^{\prime}$ at different temperature or by blackbody radiation $\mathrm{BB}$. In the absence of initial coherences in the system eigenbasis, we show in situation $(i)$ that, although time dependent off-diagonal elements could be detected, they are unrelated to the dynamics of the diagonal terms. In situation (ii), coherences having been initially generated in $\mathrm{S}$ by TB contribute naturally to the dynamics of the populations of S toward the new steady state.

\section{COHERENCES AND DYNAMICS TOWARD THERMALIZATION}

The process described above is not a particular feature of light-harvesting systems, but is a generic feature of open quantum systems and is therefore ubiquitous. In order to appreciate this in detail, and to extract the relevant features of the underlying physical situation, we consider a model: a harmonic oscillator immersed in a dissipative environment TB [21-23]. Although an idealization, it encompasses a reasonable description of a wide variety of objects in nature such as low energy vibrational molecular modes, in addition to artificial ones like nanomechanical oscillators, optical and microwave cavities, and movable mirrors [24]. The Hamiltonian of the system+environment can be written as $\hat{H}=\hat{H}_{\mathrm{S}}+\hat{H}_{\mathrm{TB}}+\hat{H}_{\mathrm{ST}}$, where $\hat{H}_{\mathrm{S}}$ is the Hamiltonian of the unperturbed oscillator, $\hat{H}_{\mathrm{TB}}$ is the Hamiltonian of the thermal bath TB, and $\hat{H}_{\mathrm{ST}}$ describes the interaction of the system with TB. In particular, we choose

$$
\hat{H}=\hat{H}_{\mathrm{S}}+\sum_{j}^{\infty} \frac{\hat{p}_{j}^{2}}{2 m_{j}}+\frac{m_{j} \omega_{j}^{2}}{2}\left(\hat{q}_{j}-\frac{c_{j} \hat{q}_{x}}{m_{j} \omega_{j}^{2}}\right)^{2},
$$

and $\hat{H}_{\mathrm{S}}=\frac{1}{2 m} \hat{p}_{x}^{2}+\frac{m \omega_{0}^{2}}{2} \hat{q}_{x}^{2}$, where $m$ and $\omega_{0}$ are the natural mass and frequency of the oscillator while $\hat{p}_{x}$ and $\hat{q}_{x}$ denote its canonical conjugate momentum and position coordinates. Momenta and coordinates of bath mode $j$ are denoted $\hat{p}_{j}$ and $\hat{q}_{j}$ and $c_{j}$ denotes the coupling term to the $j$-th mode. In Fig. 1, we have depicted the physical situation.

The evolution of the system density-matrix can be analytically derived by means of the Feynman-Vernon influence functional approach [21, 22, 25]. This approach allows exploring any regime, low or high temperature, strong or weak damping, etc. (For details on this approach see Refs. [21, 22, 25, 26] or Ref. [27].) At this point, we assume that initially the density matrix of $\mathrm{S}$ and $\mathrm{TB}$ factorizes, i.e., $\hat{\rho}(0)=\hat{\rho}_{\mathrm{S}}(0) \otimes \hat{\rho}_{\mathrm{TB}}(0)$ and additionally that each mode of the bath is at thermal equilibrium at

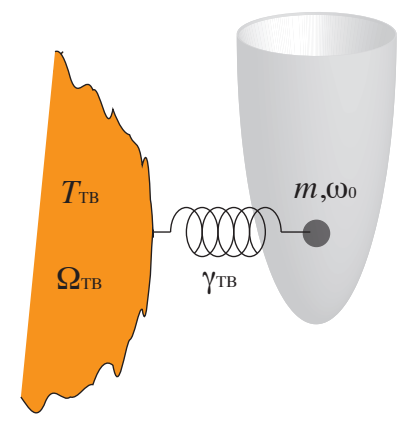

FIG. 1. The system S, initially described by $\hat{\rho}_{\mathrm{S}}(0)$, is coupled to the thermal bath TB at temperature $T_{\mathrm{TB}}$ and frequency cutoff $\Omega_{\mathrm{TB}} \cdot \gamma_{\mathrm{TB}}$ denotes the strength of the cooling.

the same temperature $T_{\mathrm{TB}}[21,22,25]$.

Within this framework, the time evolution of the density matrix is given by

$$
\begin{aligned}
& \left\langle q_{+}^{\prime \prime}\left|\hat{\rho}_{\mathrm{S}}(t)\right| q_{-}^{\prime \prime}\right\rangle=\int \mathrm{d} q_{+}^{\prime} \mathrm{d} q_{-}^{\prime} \\
& \quad \times J\left(q_{+}^{\prime \prime}, q_{-}^{\prime \prime}, t, q_{+}^{\prime}, q_{-}^{\prime}, 0\right)\left\langle q_{+}^{\prime}\left|\hat{\rho}_{\mathrm{S}}(0)\right| q_{-}^{\prime}\right\rangle
\end{aligned}
$$

where the $q$ label denotes the system coordinate representation, $J\left(q_{+}^{\prime \prime}, q_{-}^{\prime \prime}, t, q_{+}^{\prime}, q_{-}^{\prime}, 0\right)$ is the influence functional, which is given in terms of a path integral after tracing out the environmental degrees of freedom (see Refs. [21, 22, 25, 26] or Ref. [27]). This solution does not provide direct insight into the dynamics in the eigenbasis of $\hat{H}_{\mathrm{S}}$. However, the solution given in Refs. [21, 22] can be analytically transformed into the eigenbasis of $\hat{H}_{\mathrm{S}}$ (denoted by $\{|n\rangle\}$ with eigenvalues $\left\{E_{n}\right\}$ ) giving the following expression:

$$
\begin{aligned}
\left\langle n\left|\hat{\rho}_{\mathrm{S}}(t)\right| m\right\rangle & =\sum_{\nu} J_{n m ; \nu \nu}(t)\left\langle\nu\left|\hat{\rho}_{\mathrm{S}}(0)\right| \nu\right\rangle \\
& +\sum_{\nu \neq \mu} J_{n m ; \nu \mu}(t)\left\langle\nu\left|\hat{\rho}_{\mathrm{S}}(0)\right| \mu\right\rangle,
\end{aligned}
$$

where $J_{n m ; \nu \mu}(t)$ is the influence functional in the energy basis representation, i.e.,

$$
\begin{aligned}
J_{n m ; \nu \mu}(t) & =\int \mathrm{d} q_{+}^{\prime \prime} \mathrm{d} q_{-}^{\prime \prime} \mathrm{d} q_{+}^{\prime} \mathrm{d} q_{-}^{\prime} J\left(q_{+}^{\prime \prime}, q_{-}^{\prime \prime}, t, q_{+}^{\prime}, q_{-}^{\prime}, 0\right) \\
& \times\left\langle n \mid q_{+}^{\prime \prime}\right\rangle\left\langle q_{-}^{\prime \prime} \mid m\right\rangle\left\langle q_{+}^{\prime} \mid \nu\right\rangle\left\langle\mu \mid q_{-}^{\prime}\right\rangle .
\end{aligned}
$$

The result provides a remarkable linear map between the initial system state and the final system state that (see below) even holds in the case of initial system-bath correlations.

Of particular interest is the interplay between the diagonal elements of the density matrix (state populations) and the off-diagonal elements (coherences). In absence of coupling to the environment the kernel $J_{n m ; \nu \mu}(t)$ reduces to $J_{n m ; \nu \mu}(t)=\mathrm{e}^{-\mathrm{i}\left(E_{m}-E_{n}\right) t / \hbar} \delta_{n \nu} \delta_{m \mu}$. The role of the $\delta_{n \nu} \delta_{m \beta}$ is twofold: it prevents the transfer of initial population from $\left\langle\nu\left|\hat{\rho}_{\mathrm{S}}(0)\right| \nu\right\rangle$ to $\left\langle n\left|\hat{\rho}_{\mathrm{S}}(t)\right| n\right\rangle$ and addition- 
ally, it eliminates any effect of initial off-diagonal elements on the populations (i.e., the diagonal elements). By contrast, in the presence of coupling to the environment, the overlap of the system eigenstates generates "new routes" for affecting populations, provided by terms of the type $J_{n n ; \nu \nu}(t)$. These terms transfer the initial population $\left\langle\nu\left|\hat{\rho}_{\mathrm{S}}(0)\right| \nu\right\rangle$ to $\left\langle n\left|\hat{\rho}_{\mathrm{S}}(t)\right| n\right\rangle$ at time $t$. Additionally, any initial off-diagonal elements contribute to the time dependence of the populations through the nonzero $J_{n n ; \nu \mu}$ terms.

For the system-bath bilinear coupling in Fig. 1, the influence and nature of the bath is determined by a spectral density $J(\omega)[21,22,25]$, which can be expressed in terms of the bath modes parameters: $J(\omega)=$ $\pi \sum \frac{c_{j}^{2}}{2 m_{j} \omega_{j}} \delta\left(\omega-\omega_{j}\right)$. In this case, we assume an nonMarkovian Ohmic spectral density

$$
J(\omega)=m \gamma_{\mathrm{TB}} \omega \Omega_{\mathrm{TB}}^{2} /\left(\Omega_{\mathrm{TB}}^{2}+\omega^{2}\right),
$$

where $\gamma_{\mathrm{TB}}$ is the strength coupling constant to the thermal bath and $\Omega_{\mathrm{TB}}$ is a frequency cutoff. This spectral density generates the following damping kernel $m \gamma(t)=\sum_{j} \frac{c_{j}^{2}}{2 m_{j} \omega_{j}^{2}} \cos \left(\omega_{j} t\right)=2 \int_{0}^{\infty} \frac{\mathrm{d} \omega}{\pi} \frac{J_{\mathrm{TB}}(\omega)}{\omega} \cos (\omega t)=$ $\gamma_{\mathrm{TB}} \Omega_{\mathrm{TB}} \exp \left(-\Omega_{\mathrm{TB}}|t|\right)$. This kernel is responsible for the relaxation process and describes, roughly, the rate at which energy is transferred to the bath. In the limit when the cutoff frequency $\Omega_{\mathrm{TB}}$ tends to infinity, $\gamma(t) \rightarrow 2 \gamma_{\mathrm{TB}} \delta(t)$, which corresponds to Markovian Ohmic dissipation.

If initially $\hat{\rho}_{\mathrm{S}}(0)=\sum_{n}|n\rangle\langle n|$, i.e., there is no initial coherence, the second sum in Eq. (3) vanishes and the time evolution of the diagonal terms depends exclusively on their initial values. For example, for the particular case when $\hat{\rho}_{\mathrm{S}}(0)=|0\rangle\langle 0|$, the subsequent time evolution of the diagonal elements of the density matrix can be expressed simply by

$$
\left\langle n\left|\hat{\rho}_{\mathrm{S}}(t)\right| n\right\rangle=J_{n n ; 00}(t) .
$$

In Fig. 2, we have depicted $J_{n n ; 00}(t)$ for some values of $n$. By contrast to the unitary case where $J_{n n ; 00}(t)=\delta_{n 0} \delta_{n 0}$, here we have the possibility of populating different energy levels. As expected for a thermalizing system, the amount of population transfer increases with increasing temperature (see Fig. 2). So, it is clear that the role of $J_{n n ; 00}(t)$ is transferring of population from $\left\langle 0\left|\hat{\rho}_{S}\right| 0\right\rangle$ to other eigenstates of $\mathrm{S}$. This is a natural consequence of the fact that the spectrum of open quantum systems is broadened [21, 22, 25, 26], so excitation of single energy levels is not possible because of the overlap. These environmentally induced "new routes" for population transfer from different eigenstates have been extensively exploited in the context of biological systems [28, 29]. Here we can see that they appear mediating thermal activation, with the process being incoherent in nature.

Remarkably, see Fig. 3, this population transfer is accompanied by the generation of off-diagonal terms of

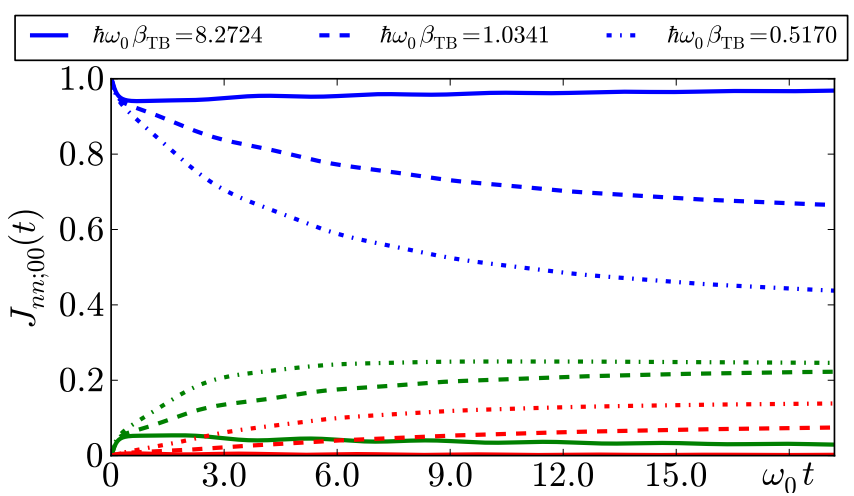

FIG. 2. (Color online). Time evolution of $J_{00 ; 00}(t)$ (blue curves), $J_{11 ; 00}(t)$ (green curves) and $J_{22 ; 00}(t)$ (red curves). Results are for $\gamma_{\mathrm{TB}}=0.1 \omega_{0}, \Omega_{\mathrm{TB}}=20 \omega_{0}$ and $\hbar \omega_{0} / k_{\mathrm{B}} T_{\mathrm{TB}}=$ 8.2724 (continuous curves), $\hbar \omega_{0} / k_{\mathrm{B}} T_{\mathrm{TB}}=1.0341$ (dashed curves) and $\hbar \omega_{0} / k_{\mathrm{B}} T_{\mathrm{TB}}=0.5179$ (dot-dashed curves).

the density matrix, $\left\langle n\left|\rho_{\mathrm{S}}(t)\right| m\right\rangle=J_{n m ; 00}(t)$. By contrast to the diagonal terms, the asymptotic value of these off-diagonal terms are seen to increase with decreasing temperature and hence they become relevant at low temperature. For an harmonic bath, low temperature is accompanied by non-Markovian decoherence dynamics of the system. More importantly, the time decay seen in Fig. 3 is not related to decaying coherences because initially there was no coherence in the density matrix. Rather, they reflect the fact that in the effective basis, the populations are changing to reach the thermal state. It is worth noticing that these off-diagonal terms cannot be related to the existence of a coherent superposition of states, but to the overlapping of energy eigenstates induced by the incoherent effect of the bath.

Note, significantly, that terms like $J_{n m ; 00}$ shown in Fig. 3 do not go to zero at long times. This implies that the associated [See Eq. (3)] $\left\langle n\left|\hat{\rho}_{\mathrm{S}}(t)\right| m\right\rangle$ matrix element assumes a long-time constant nonzero value.

In order to understand the nature of these off-diagonal terms, note that the density matrix is a double sided object, which means that we can interpret $J_{n m ; 00}(t)$ as the influence-functional element associated with transitions from $|0\rangle \rightarrow|n\rangle$ and $\langle m| \leftarrow\langle 0|$ mediated by thermal activation (similar terms arise in optical nonlinear response [30]). At high temperature, $\hbar \omega_{0} \beta_{\mathrm{TB}} \gg 1$ and $\frac{1}{2} \hbar \gamma_{\mathrm{TB}} \beta_{\mathrm{TB}} \gg 1$, we have typically "symmetric" transitions, which are associated with transitions of the type $|0\rangle \rightarrow|n\rangle$ and $\langle n| \leftarrow\langle 0|$. "Asymmetric" transitions, associated with transitions of the type $|0\rangle \rightarrow|n\rangle$ and $\langle m| \leftarrow\langle 0|$, are less probable; however, they are enhanced at low temperature, $\hbar \omega_{0} \beta_{\mathrm{TB}} \ll 1$ and $\frac{1}{2} \hbar \gamma_{\mathrm{TB}} \beta_{\mathrm{TB}} \ll 1$ as seen in Fig. 3 .

The fact that these off-diagonal terms survive at equilibrium, points out the possibility of deviations from "canonical typicality" [31] (i.e., diagonal equilibrium state in the system energy eigenbasis) at low temperature [32]. From our discussion, we can note that these off-diagonal terms are not attributable to interfering processes, but rather 

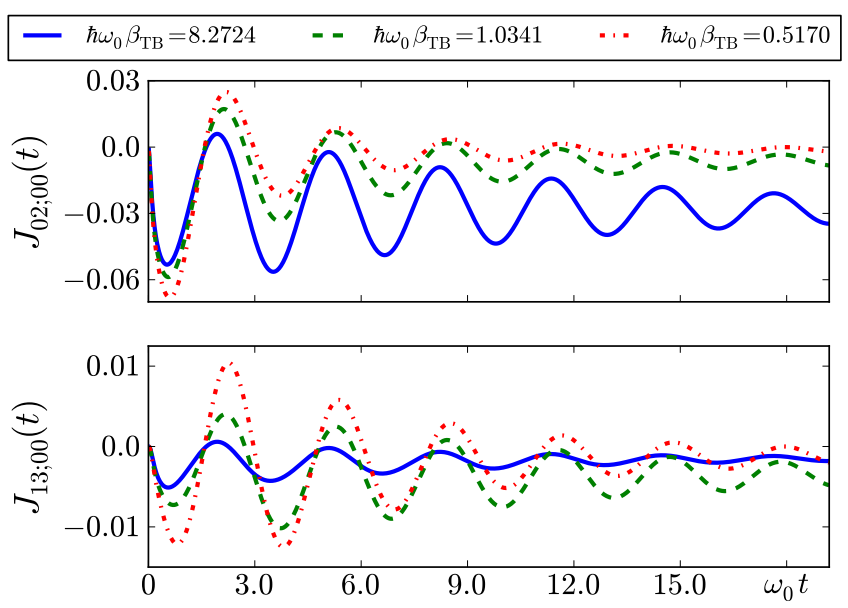

FIG. 3. (Color online). Time evolution of $J_{02 ; 00}(t)$ (upper panel) and $J_{13 ; 00}(t)$ (lower panel). Results are for $\gamma_{\mathrm{TB}}=$ $0.1 \omega_{0}, \Omega_{\mathrm{TB}}=20 \omega_{0}$ and $\hbar \omega_{0} / k_{\mathrm{B}} T_{\mathrm{TB}}=8.2724$ (continuous blue curves), $\hbar \omega_{0} / k_{\mathrm{B}} T_{\mathrm{TB}}=1.0341$ (dashed green curves) and $\hbar \omega_{0} / k_{\mathrm{B}} T_{\mathrm{TB}}=0.5179$ (dot-dashed red curves).

are part of thermal activation induced by the coupling to the bath, so they can take constant nonzero values at equilibrium rather than just vanishing. Based on this description, we should not call them coherences, but just static off-diagonal terms. However, these have been called stationary coherences, as noted in Sec. II and are simply static manifestations at equilibrium, of the system-bath coupling.

At equilibrium, the magnitude of the off-diagonal terms in the system density matrix can be evaluated from the equilibrium density matrix [33], i.e., $\hat{\rho}_{\beta}=$ $\mathcal{Z}_{\beta_{\mathrm{TB}}}^{-1} \operatorname{Tr}_{\mathrm{TB}} \exp \left[-\beta_{\mathrm{TB}}\left(\hat{H}_{\mathrm{S}}+\hat{H}_{\mathrm{ST}}+\hat{H}_{\mathrm{TB}}\right)\right]$, where $\mathcal{Z}_{\beta_{\mathrm{TB}}}$ is a normalization factor and $\operatorname{Tr}_{\mathrm{TB}}$ denotes the trace over the bath. For this particular case, we can introduce the effective Hamiltonian $\hat{H}_{\text {eff }}=\frac{1}{2 m_{\text {eff }}} \hat{p}_{x}^{2}+\frac{1}{2} m_{\text {eff }} \omega_{\text {eff }}^{2} \hat{q}_{x}^{2}$, with the effective mass $m_{\text {eff }}=\omega_{\text {eff }}^{-1} \sqrt{\left\langle p^{2}\right\rangle\left\langle q^{2}\right\rangle^{-1}}$, and the effective frequency $\omega_{\text {eff }}=2\left(\hbar \beta_{\mathrm{TB}}\right)^{-1} \operatorname{arccoth}\left(\frac{2}{\hbar} \sqrt{\left\langle p^{2}\right\rangle\left\langle q^{2}\right\rangle}\right)$, while $\left\langle q^{2}\right\rangle$ and $\left\langle p^{2}\right\rangle$ being the equilibrium variances [22, 33]. This allows expressing $\hat{\rho}_{\beta}$ as [33]

$$
\hat{\rho}_{\beta}=Z_{\beta_{\mathrm{TB}}}^{-1} \sum_{n=0}^{\infty} \exp \left(-E_{n_{\beta}} \beta_{\mathrm{TB}}\right)\left|n_{\beta}\right\rangle\left\langle n_{\beta}\right|,
$$

with $E_{n_{\beta}}=\hbar \omega_{\text {eff }}\left(n_{\beta}+\frac{1}{2}\right)$ being the eigenvalues and $\left|n_{\beta}\right\rangle$ the eigenstates of the effective Hamiltonian $\hat{H}_{\text {eff }}$ and $Z_{\beta_{\mathrm{TB}}}$ is the generalized partition function [33]. At high temperature, $\hbar \omega_{0} \beta_{\mathrm{TB}} \ll 1$ and $\frac{1}{2} \hbar \gamma_{\mathrm{TB}} \beta_{\mathrm{TB}} \ll 1, m_{\text {eff }}$ and $\omega_{\text {eff }}$ approaches their bare values $m$ and $\omega_{0}$, respectively, and $\hat{\rho}_{\beta}$ tends to the canonical distribution [33, 34] (with no off-diagonal terms in the system energy eigenbasis). At low temperatures, $\hbar \omega_{0} \beta_{\mathrm{TB}} \gg 1$ and $\frac{1}{2} \hbar \gamma_{\mathrm{TB}} \beta_{\mathrm{TB}} \gg 1$, they undergo to strong deviation due to damping [33, 34].

Since we are interested in natural processes, we next

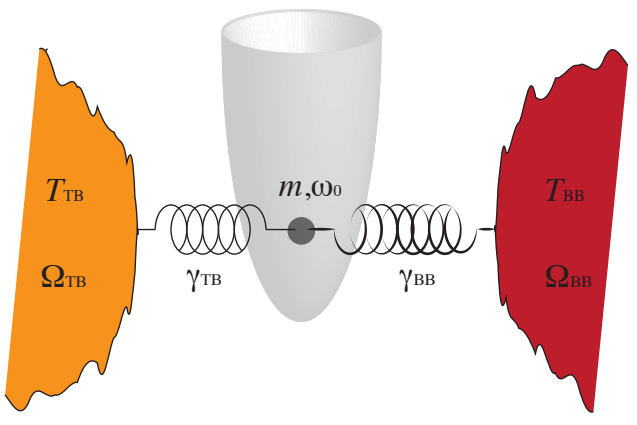

FIG. 4. After thermalizing with TB, the system $\mathrm{S}$ is put in contact with a second thermal bath $\mathrm{TB}^{\prime}$ (or $\mathrm{BB}$, as in the plot) at different temperature and different coupling constant.

consider excitation of the equilibrated system $\mathrm{S}$ by $(i)$ a second thermal bath $\mathrm{TB}^{\prime}$ of the same nature as TB but at a different temperature and different coupling constant, and (ii) by blackbody radiation, denoted BB. For example, in the particular case of biological processes, e.g., in electronic energy transfer in photosynthetic complexes $[1,10]$, the coupling to the environment is strong [35] and the effective temperature is low $[16,17]$. So, a question immediately follows: do these off-diagonal terms play any relevant role in any subsequent dynamics of these systems? More specifically, in what way do they contribute to any dynamics arising from subsequent perturbation?

\section{DYNAMICS IN THE PRESENCE OF A SECOND THERMAL BATH}

Equilibration of a system at temperature $T_{\mathrm{TB}}$, with another bath at temperature $T_{\mathrm{TB}^{\prime}}$ is of general interest. For example, for biological systems, thermal activation of biological processes by temperature changes can be found, e.g., in the context of transport of Ca ions through membranes [9]. To examine such processes, after thermalizing with TB, we couple the oscillator to a second dissipative environment $\mathrm{TB}^{\prime}$. The Hamiltonian of the system can now be written as

$$
\hat{H}=\hat{H}_{\mathrm{S}}+\hat{H}_{\mathrm{TB}}+\hat{H}_{\mathrm{TB}^{\prime}}+\hat{H}_{\mathrm{ST}}+\hat{H}_{\mathrm{ST}^{\prime}},
$$

where $\hat{H}_{\mathrm{TB}^{\prime}}$ is the Hamiltonian describing the second thermal bath $\mathrm{TB}^{\prime}$, while $\hat{H}_{\mathrm{ST}^{\prime}}$ describes the interaction of the system with $\mathrm{TB}^{\prime}$. As in the previous case, we choose $\hat{H}_{\mathrm{TB}^{\prime}}$ as being composed of a set of harmonic oscillators, so $\hat{H}=\hat{H}_{\mathrm{S}}+\sum_{j, k}^{\infty, 2} \frac{\hat{p}_{j, k}^{2}}{2 m_{j, k}}+\frac{m_{j, k} \omega_{j, k}^{2}}{2}\left(\hat{q}_{j, k}-\frac{c_{j, k} \hat{q}_{x}}{m_{j, k} \omega_{j, k}^{2}}\right)^{2}$. Note that the coupling is of the system $\mathrm{S}$ to the heat bath $\mathrm{TB}$ and of the system to the heat bath $\mathrm{TB}^{\prime}$, as shown in Fig. 4. There is no direct coupling between TB and $\mathrm{TB}^{\prime}$.

The resultant evolution of the system density-matrix can also be analytically obtained by using the influence functional approach [27]. The evolution is of the form in Eq. 3, but with $\hat{\rho}_{\mathrm{S}}(0)=\hat{\rho}_{\beta}$ and the $J_{n m ; \nu \mu}(t)$ 's containing 
information about the effect of the initial correlation on the subsequent dynamics.

In our approach, we have exact analytical access to every contribution to the dynamics. By contrast, for example, when applying the secular approximation as in Ref. [6], the contribution from the off-diagonal terms $\nu \neq \mu$ is ignored.

It is important to note that in this case the initial condition is $\hat{\rho}(0)=\hat{\rho}_{\mathrm{S}+\mathrm{TB}}(0) \otimes \hat{\rho}_{\mathrm{TB}^{\prime}}(0)$, where $\hat{\rho}_{\mathrm{S}+\mathrm{TB}}(0)$ is the equilibrium density operator of $(\mathrm{S}+\mathrm{TB})$, and $\hat{\rho}_{\mathrm{TB}^{\prime}}(0)$ denotes the thermal density operator of the second bath at temperature $T_{\mathrm{TB}^{\prime}}$. We describe the effect of the baths using, for both $\mathrm{TB}$ and $\mathrm{TB}^{\prime}$, the functional form given in Eq. (5). In the absence of $\mathrm{TB}^{\prime}$, the overall $(\mathrm{S}+\mathrm{TB})$ is time-independent, as it should be thermal equilibrium $[22,36,37]$.

During this second equilibration step, the off-diagonal elements of the system density matrix that were generated during the thermalization step affect the population dynamics (the diagonal terms) because they now enter in the initial density matrix [see Eq. (3)].
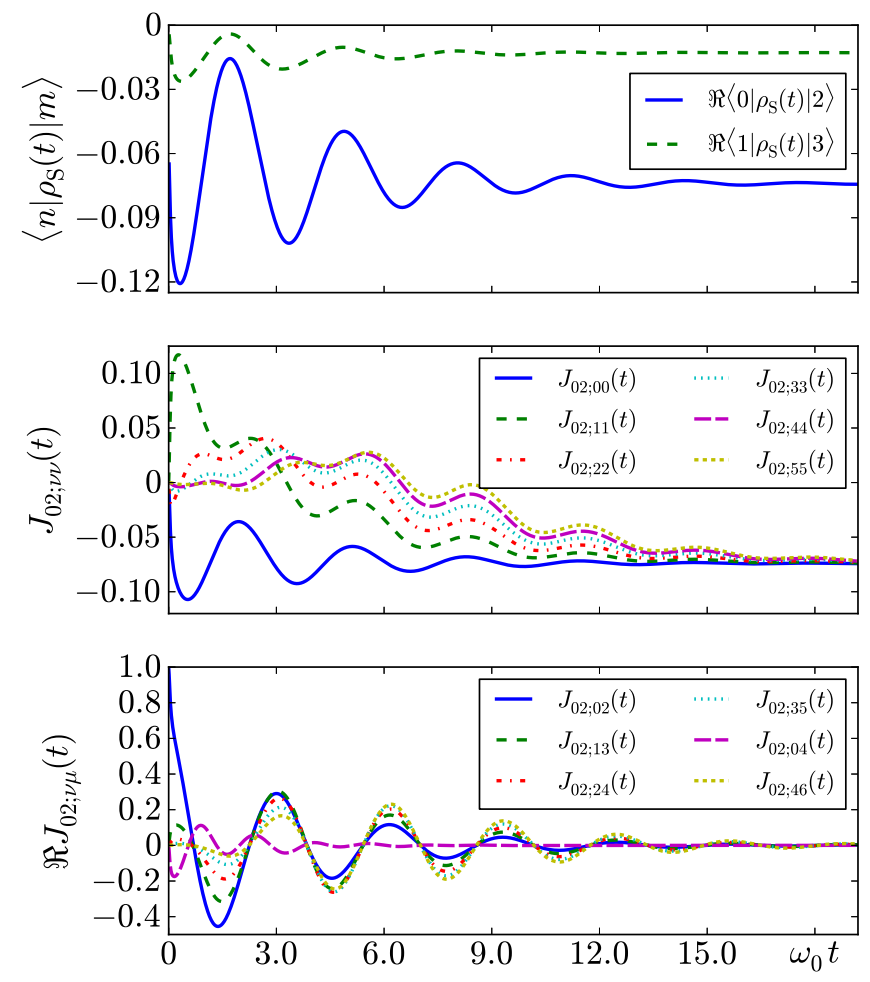

FIG. 5. (Color online). Upper panel: exact time evolution of $\left\langle 0\left|\hat{\rho}_{\mathrm{S}}\right| 2\right\rangle$ and $\left\langle 1\left|\hat{\rho}_{\mathrm{S}}\right| 3\right\rangle$ with $\hbar \omega_{0} / k_{\mathrm{B}} T_{\mathrm{TB}}=8.2724, \gamma_{\mathrm{TB}}=0.1 \omega_{0}$, $\Omega_{\mathrm{TB}^{\prime}}=20 \omega_{0}, T_{\mathrm{TB}^{\prime}}=2 T_{\mathrm{TB}}, \gamma_{\mathrm{TB}}=2 \gamma_{\mathrm{TB}}$ and $\Omega_{\mathrm{TB}^{\prime}}=2 \Omega_{\mathrm{TB}}$. Lower panels: time evolution of $J_{02 ; \nu \mu}$ for some values of $\nu$ and $\mu$.

In the upper panel of Fig. 5, we present the time evolution of $\left\langle 0\left|\hat{\rho}_{\mathrm{S}}(t)\right| 2\right\rangle$ and $\left\langle 1\left|\hat{\rho}_{\mathrm{S}}(t)\right| 3\right\rangle$ for the conditions indicated in the figure caption. The off-diagonal terms are seen to reach the same order of magnitude as the populations (not shown), i.e. $10^{-1}$. Time evolution of, e.g., $\left\langle 0\left|\hat{\rho}_{\mathrm{S}}(t)\right| 2\right\rangle$ is affected by terms of the type $J_{02, \nu \nu}$ and $J_{02, \nu \mu}$. In particular, population transfer is assisted by $J_{02, \nu \nu}$ and decay of the initial coherences is mediate by $J_{02, \nu \mu}$. In the central and lower panels of Fig. 5 , we have depicted $J_{02, \nu \nu}$ and $J_{02, \nu \mu}$, respectively, for various values of $\nu$ and $\mu$. There we can see that while $J_{02, \nu \nu}$ reach an asymptotic finite value, $J_{02, \nu \mu}$ goes to zero as time evolves. Hence, it is clear that terms like $J_{02, \nu \mu}$ are qualitatively different from $J_{02, \nu \nu}$ type terms. The former are related to the decay of quantum coherences while the latter are related to population transfer during the equilibration.

Figure 6 shows the time evolution of the ground state population $\left\langle 0\left|\hat{\rho}_{\mathrm{S}}(t)\right| 0\right\rangle$. The exact evolution is depicted by the continuous blue curve, the evolution disregarding any correlation with TB [i.e., using the canonical distribution for $\left.\hat{\rho}_{\mathrm{S}}(0)\right]$ is shown by the dot-dashed red curve and the evolution neglecting the off-diagonal terms (i.e. the secular approximation) in Eq. (3) is shown by the dashed green curve. The fact that the dynamics in these
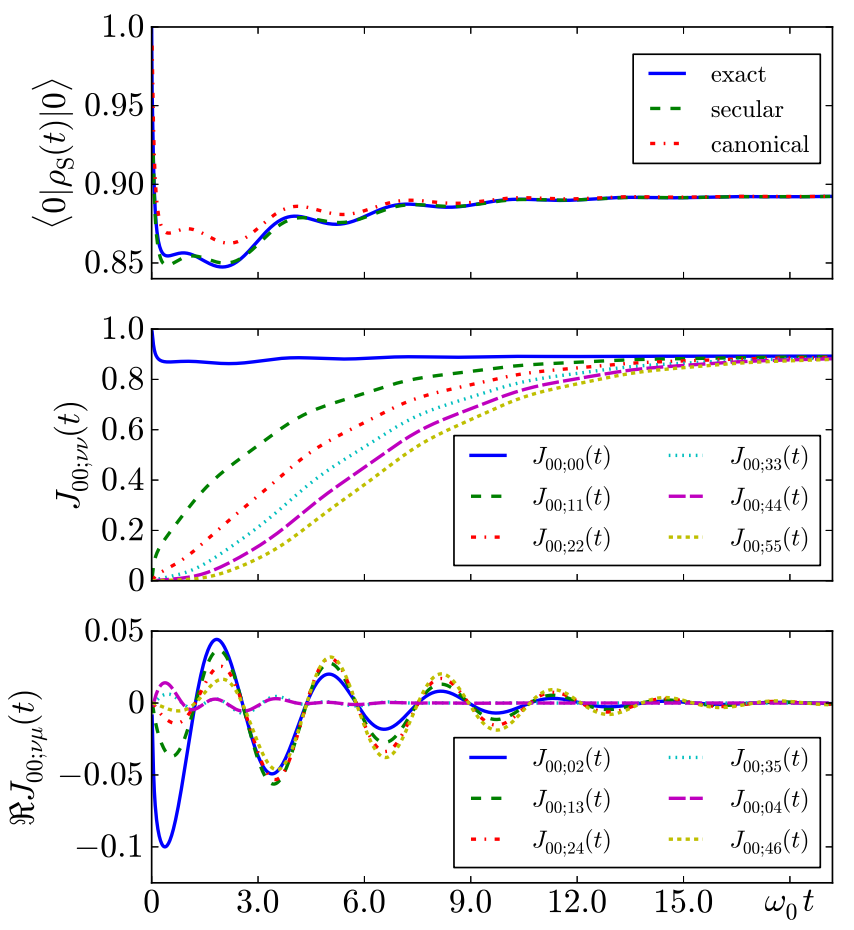

FIG. 6. (Color online). Upper panel: exact (continuous blue curve), secular-approximated (dashed green curve) time evolution of $\left\langle 0\left|\hat{\rho}_{\mathrm{S}}\right| 0\right\rangle$. The evolution using the canonical distribution as the initial state is depicted by the dot-dashed red curve. Lower panels: time evolution $J_{00 ; \nu \mu}$ for some values of $\nu$ and $\mu$. Parameters as in Fig. 5.

three cases is practically the same shows that, although off-diagonal terms are present in Fig. 5, they need not imply a significant contribution to the dynamics between eigenstates. That is, the presence of off-diagonal terms in the density matrix of open quantum systems in the system eigenstate representation is not necessarily an indicator 
of coherent effects. The lower panels in Fig. 6 show some of the non-vanishing $J_{00 ; \nu \mu}(t)$ elements.

It is worth noting that off-diagonal terms generated by the presence of the second bath cannot manipulate the dynamics of the populations by themselves. This can be seen when projecting in the basis in which $\hat{\rho}_{\beta}$ is diagonal, i.e. $\left\langle n_{\beta}\left|\hat{\rho}_{\mathrm{S}}(t)\right| n_{\beta}\right\rangle=\sum_{\nu_{\beta}} J_{n_{\beta} n_{\beta} ; \nu_{\beta} \nu_{\beta}}(t)\left\langle\nu_{\beta}\left|\hat{\rho}_{\beta}\right| \nu_{\beta}\right\rangle$. This means that although off-diagonal terms are generated (see Fig. 5), they do not participate in the evolution of the populations. Only the combined action of the thermal baths, applied sequentially, can lead to the possibility of altering the dynamics of the populations. Connecting the system to the baths, either separately or simultaneously only leads to excitation of the system.

\section{DYNAMICS INDUCED BY BLACKBODY RADIATION}

Consider now the case where the second thermal bath $\mathrm{TB}^{\prime}$ is replaced by blackbody radiation (BB). Here, a charged harmonic oscillator is immersed in a dissipative environment TB and coupled via dipole approximation to blackbody radiation BB. This provides a generic model for a wide variety of objects such as atoms, ions, electrons, molecules in equilibrium, subjected to blackbody irradiation. Qualitative results for this case are particularly relevant for natural light incident on biomolecules.

The Hamiltonian of the total system is now of the form of Eq. (8) where $\hat{H}_{\mathrm{B}}$ is the Hamiltonian describing the radiation field and $\hat{H}_{\mathrm{SB}^{\prime}}$ describes the interaction of the system with the blackbody radiation. In the dipole approximation, the Hamiltonian for an oscillator interacting with a radiation field and coupled linearly to its surrounding environment is

$$
\begin{aligned}
& \hat{H}=\frac{1}{2 m}\left(\hat{p}_{x}-\frac{e}{c} \hat{A}_{x}\right)^{2}+\frac{m \omega_{0}^{2}}{2} \hat{q}_{x}^{2}+\sum_{j}\left[\frac{\hat{p}_{j}^{2}}{2 m_{j}}\right. \\
& \left.+\frac{m_{j} \omega_{j}^{2}}{2}\left(\hat{q}_{j}-\frac{c_{j} \hat{q}_{x}}{m_{j} \omega_{j}^{2}}\right)^{2}\right]+\sum_{\mathbf{k}, s} \hbar c k\left(\hat{a}_{\mathbf{k}, s}^{\dagger} \hat{a}_{\mathbf{k}, s}+\frac{1}{2}\right),
\end{aligned}
$$

where $e / c$ is the coupling constant to the radiation, $\hat{a}_{\mathbf{k}, s}$ and $\hat{a}_{\mathbf{k}, s}^{\dagger}$ are the annihilation and creation operators of the field mode of momentum $\mathbf{k}$ and polarization $s$. The vector potential is given by

$$
\hat{A}_{x}=\sum_{\mathbf{k}, s}\left[\frac{h c}{k V}\right]^{\frac{1}{2}}\left[f_{k}^{*} \hat{a}_{\mathbf{k}, s} \mathbf{e}_{\mathbf{k}, s} \cdot \mathbf{q}+f_{k} \hat{a}_{\mathbf{k}, s}^{\dagger} \mathbf{e}_{\mathbf{k}, s}^{*} \cdot \mathbf{q}\right],
$$

where $\mathbf{e}$ is the polarization vector, $V$ is the volume of the cavity and $f_{k}$ is the electron form-factor (Fourier transform of the charge distribution) which incorporates the electron structure [23].

By defining $m_{\mathbf{k}}=4 \pi e^{2} f_{k}^{2} /\left(\omega_{\mathbf{k}} V\right)$ and $a_{\mathbf{k}, s}=$ $\left(m_{\mathbf{k}} \omega_{\mathbf{k}} q_{\mathbf{k}, s}+\mathrm{i} p_{\mathbf{k}, s}\right) / \sqrt{2 m_{\mathbf{k}} \hbar \omega_{\mathbf{k}}}$, and applying the PowerZienau's transformation (see Ref. [27] for details) we can rewrite Eq. (9) as

$$
\begin{aligned}
\hat{H} & =\frac{1}{2 m} \hat{p}_{x}^{2}+\frac{1}{2} m \omega_{0}^{2} \hat{q}_{x}^{2} \\
& +\sum_{\mathbf{k}, s} \frac{1}{2 m_{\mathbf{k}}}\left(\hat{p}_{\mathbf{k}, s}+m_{\mathbf{k}} \omega_{\mathbf{k}} \hat{q}_{x}\right)^{2}+\frac{1}{2} m_{\mathbf{k}} \omega_{\mathbf{k}, s}^{2} \hat{q}_{\mathbf{k}, s}^{2} \\
& +\sum_{j} \frac{\hat{p}_{j}^{2}}{2 m_{j}}+\frac{1}{2} m_{j} \omega_{j}^{2}\left(\hat{q}_{j}-\frac{c_{j} \hat{q}_{x}}{m_{j} \omega_{j}^{2}}\right)^{2},
\end{aligned}
$$

where the oscillator is seen to be coupled to the momentum coordinate $\hat{p}_{\mathbf{k}, s}$. Since $\hat{E}_{x}=-\partial \hat{A}_{x} / \partial t$ and based on the definition of the annihilation and creation operators (see above), one can show that this momentum coupling is equivalent to the oscillator being coupled to the electric field of the radiation with

$$
\hat{E}_{x}=\mathrm{i} \sum_{\mathbf{k}, s}\left(\frac{h c^{3}}{V}\right)^{\frac{1}{2}}\left(f_{k}^{*} \hat{a}_{\mathbf{k}, s} \mathbf{e}_{\mathbf{k}, s} \cdot \mathbf{q}+f_{k} \hat{a}_{\mathbf{k}, s}^{\dagger} \mathbf{e}_{\mathbf{k}, s}^{*} \cdot \mathbf{q}\right) .
$$

In order to describe the action of the blackbody radiation, we need to consider, as in the previous case, that the modes in the cavity are thermally populated (for more details see Ref. [27]), i.e., each mode is characterized by an incoherent density operator. This feature introduces the incoherent nature of the radiation considered in this work. Based on this description, we expect that the blackbody radiation generates an incoherent evolution in contrast with the coherent evolution induced by laser pulses.

Interestingly, the thermal fluctuations generated by the blackbody radiation are characterized by a two-point electric-field correlation function $\left\langle\hat{E}_{x}\left(t^{\prime \prime}\right) \hat{E}_{x}\left(t^{\prime}\right)\right\rangle$ that is not $\delta$-correlated. This reveals the intrinsic non-Markovian character of the radiation from both a statistical viewpoint $[23,27,38]$ and from the optics point of view [39]. The correlation time $\tau_{\mathrm{BB}}^{\mathrm{c}}$ of the randomly fluctuating electric field can be calculated from the thermal time $\tau_{\mathrm{BB}}^{\text {th }}=$ $\hbar / k_{\mathrm{B}} T_{\mathrm{BB}}$ [39] and is expressed roughly as $\tau_{\mathrm{BB}}^{\mathrm{c}} \sim \tau_{\mathrm{BB}}^{\mathrm{th}}$. For blackbody radiation at $T_{\mathrm{BB}}=300 \mathrm{~K}, \tau_{\mathrm{BB}}^{\mathrm{c}} \sim 25.5 \mathrm{fs}$ whereas for sunlight, $T_{\mathrm{BB}}=5900 \mathrm{~K}, \tau_{\mathrm{BB}}^{\mathrm{c}} \sim 1.3 \mathrm{fs}$ and for moonlight, $T_{\mathrm{BB}}=4100 \mathrm{~K}, \tau_{\mathrm{BB}}^{\mathrm{c}} \sim 1.86 \mathrm{fs}$. Thus, for processes taking place on the order of, e.g., 1 ps (such as electronic energy transfer in photosynthetic complexes $[1,10])$ this coherence time is very short. Hence, under illumination by sunlight, the perturbation is effectively $\mathrm{CW}$ and incoherent.

From an open-quantum-system perspective, the influence of the blackbody radiation on the system is condensed in the spectral density $[23,27,38]$

$$
J_{\mathrm{BB}}(\omega)=M \tau_{\mathrm{BB}} \omega^{3} \Omega_{\mathrm{BB}}^{2} /\left(\Omega_{\mathrm{BB}}^{2}+\omega^{2}\right),
$$

where $M=m+M \tau_{\mathrm{BB}} \Omega_{\mathrm{BB}}$ is the renormalized mass, $\tau_{\mathrm{BB}}=2 e^{2} / 3 M_{\mathrm{e}} c^{3} \sim 6.24 \times 10^{-24} \mathrm{~s}$ and $\Omega_{\mathrm{BB}}$ is a frequency cutoff [23]. This spectral density generates the following dissipative kernel $\gamma_{\mathrm{BB}}(t)=$ $\tau_{\mathrm{BB}} \Omega_{\mathrm{BB}}^{2}\left[2 \delta(t)-\Omega_{\mathrm{BB}} \exp \left(-\Omega_{\mathrm{BB}}|t|\right)\right]$. Note that there is 
a fundamental limitation to the use of Eq. (12). That is, in the limit $\Omega_{\mathrm{BB}} \rightarrow \infty$, we get the surprising result, $\gamma_{\mathrm{BB}}(t)=0$, i.e. no relaxation. This corresponds to the point-electron limit $\left[f_{k}^{2}=\Omega_{\mathrm{BB}}^{2} /\left(\Omega_{\mathrm{BB}}^{2}+\omega_{k}^{2}\right)=1\right.$ in Eq. (10)] and is unphysical because even for the electron, $\Omega_{\mathrm{BB}}$ remains finite, although large. There is a natural upper limit given by $[40,41] \Omega_{\mathrm{BB}}=\tau_{\mathrm{BB}}{ }^{-1}$, which corresponds to $2 / 3$ of the time for photon to traverse the classical electron radius $\left(r_{\mathrm{cl}}^{\mathrm{e}}=2.818 \times 10^{-15} \mathrm{~m}\right)$. Beyond this natural limit, causality is violated [40] and the bare mass $m$ takes negatives values [40]. Note that one could consider another reasonable choice of form factor, e.g., $f_{k}^{2}=\Omega_{\mathrm{BB}}^{4} /\left(\Omega_{\mathrm{BB}}^{2}+\omega_{k}^{2}\right)^{2}$, corresponding to a sharper cut-off [40]. This will lead to corrections in the equation of motion of the order of $\tau_{\mathrm{BB}}$ and $\tau_{\mathrm{BB}}^{2}$, which compared with $\tau_{\mathrm{BB}}^{\mathrm{c}}$ are negligible. Thus, following [40], $f_{k}^{2}=\Omega_{\mathrm{BB}}^{2} /\left(\Omega_{\mathrm{BB}}^{2}+\omega_{k}^{2}\right)$ can be considered as an excellent approximation.

The exact analytic expression for the influence functional for this case is derived in Ref. [27]. There we show that the equations of motion are driven by a transient term that is dependent on the initial conditions and proportional to $\gamma_{\mathrm{BB}}$. This term is absent in the former case above, and is a consequence of the coupling to the momentum of the modes rather than coupling through the coordinate of the modes [cf. Eq. (11)]. So, in addition to the turn-on effect, present in the former case, in the case of incoherent excitation by blackbody radiation, we also have a driven transient term.

In order to gain insight into the strength of this transient term and of the blackbody radiation, we examine results in the limit when $\Omega_{\mathrm{BB}} \rightarrow \tau_{\mathrm{BB}}^{-1}$, and where the effect of the radiation can be estimated by a constant damping kernel given by $\gamma_{\mathrm{BB}}=\omega_{0}^{2} \tau_{\mathrm{BB}}$ [23]. Assuming a typical value of electronic molecular structure (e.g. a carbon-carbon bound) $\omega_{0}=3 \times 10^{14} \mathrm{~Hz}$, we have that $\gamma_{\mathrm{BB}}=1.8 \times 10^{-9} \omega_{0}$. In this case the excitation due to the radiation is too weak to compete with the incoherent effect of TB and, in absence of TB, the thermalization would take far too long $\left(\gamma_{\mathrm{BB}}^{-1}=1.85 \mu \mathrm{s}\right)$ to be appreciable on, e.g., a picosecond time-scale. In order to see some sort of appreciable effect on the picoseconds time scale would require a cutoff on the order of $\Omega_{\mathrm{BB}}=5 \times 10^{-6} \tau_{\mathrm{BB}}^{-1}\left(\gamma_{\mathrm{BB}}^{-1}=9 \mathrm{ps}\right)$.

As an example of the dynamics, Fig. 7 shows the time evolution of the ground state and some propagating elements $J_{00, \nu \mu}(t)$ and Fig. 8 provides the corresponding results for the first excited state. In order to see some sort of appreciable effect, we have used here the artificial value of $\Omega_{\mathrm{BB}}=5 \times 10^{-6} \tau_{\mathrm{BB}}^{-1}$ and additionally strong coupling to the environment $\gamma_{\mathrm{TB}}=10^{-1} \omega_{0}$. With these parameters, we have that in Fig. 7 and Fig. $8, T_{\mathrm{TB}}=277 \mathrm{~K}$ while $T_{\mathrm{BB}}=5900 \mathrm{~K}$. For this frequency, the time interval depicted in Fig. 7 and Fig. 8 is $\approx 128$ fs. Here, the exact evolution of $\left\langle 0\left|\hat{\rho}_{\mathrm{S}}(t)\right| 0\right\rangle$ and $\left\langle 1\left|\hat{\rho}_{\mathrm{S}}(t)\right| 1\right\rangle$ are depicted by a continuous blue line, the time evolution neglecting the off-diagonal (stationary coherent) terms initially generated by the presence of the TB is depicted using the dashed green line, and the evolution using the canonical distribution as the initial state by the dot-dashed red curve.

In the lower panels, we present some of the nonvanishing $J_{00 ; \nu \mu}(t)$ and $J_{11 ; \nu \mu}(t)$ elements. It is evident that the effect of the radiation is so weak that it generates neither off-diagonal terms nor significant changes in the populations dynamics. That is, even with the choice of excessively aggressive parameters, the effect of the radiation is negligible. Rather, the time evolution can be seen as being caused by the turn-on of the interaction followed by the subsequent relaxation of the system to equilibrium, dominated by the interaction with TB. The weak oscillations in Fig. 7 and Fig. 8, can be attributed to the transient driven term, which is strongly determined by the frequency cutoff $\Omega_{\mathrm{BB}}$. For different sets of parameters these oscillations might well be absent.

The effect of switching the interaction on $t=0$ and the time-dependent driving transient term can be also appreciated by the jump of the $J_{n m ; \nu \mu}$-terms. That is, in Fig. $7, J_{00 ; 00}$ is seen to jump from unity at $t=0$ to a different value, and $J_{00 ; 11}$ and $J_{00 ; \nu \mu}$ jump from zero to a finite value. The same applies for $J_{11 ; 00}$ and $J_{11 ; \nu \mu}$ in Fig. 8.

From Fig. 7, we can see oscillations in the population of the ground state, these oscillations are also present in, e.g., the population of the first excited state [see Fig. 8]. Since, in our "secular approximation" these oscillations are removed, according to us, this would imply that the excitation is coherent, but very short lived.

At this juncture, it is illustrative to comment on the difference between these results and those resulting when the equilibrated system (S+TB) is excited by a coherent source where it is assumed that it is the system $\mathrm{S}$ that interacts with the radiation. Given this coherent excitation (e.g. pulsed transform limited laser), the dynamical features change completely: the absorption of one photon from a coherent pulse creates a coherent superposition of energy eigenstates, and hence a time evolving state (cf. Ref. [7]). It is worth mentioning that in absence of TB, if the initial state is a pure state, then this superposition will be described by a pure state. By contrast, under the same circumstance (absence of TB), the blackbody radiation will create a incoherent superposition of states leading to a mixed state. In the presence of TB, the stationary coherent terms created during the thermalization with TB will allow us to enhance the coherent control over the populations by transferring the coherence of the pulse into the populations [42, 43].

As noted above, the parameters in the computation in Fig. 7 and Fig. 8 are artificial, and designed to show some effects due to BB. Based on naive classical arguments, this value of $\Omega_{\mathrm{BB}}$ would be equivalent to the inverse of the time needed by light to travel over around $1 \AA$. In the context of electronic energy transfer in photosynthetic complexes, where natural frequencies $\omega_{0}=6.63 \times 10^{12} \mathrm{~Hz}$ occur, then the coupling constant will be $\approx \gamma_{\mathrm{BB}}=8 \times 10^{-6} \omega_{0}$ $\left(\gamma_{\mathrm{BB}}^{-1}=19 \mathrm{~ns}\right)$. In this case the dynamics induced would be far less noticeable than the one shown in Fig. 7 and Fig. 8. That is, since $\gamma_{\mathrm{BB}}$ is smaller, then the effect on 
the relaxation will be weaker as well. Indeed, the final stationary population reached after equilibration will be far closer to that reached during the first thermalization step. Once again the response is to the turn-on of the field with rapid equilibration following mediated by the driving initial-condition-dependent-transient-term.
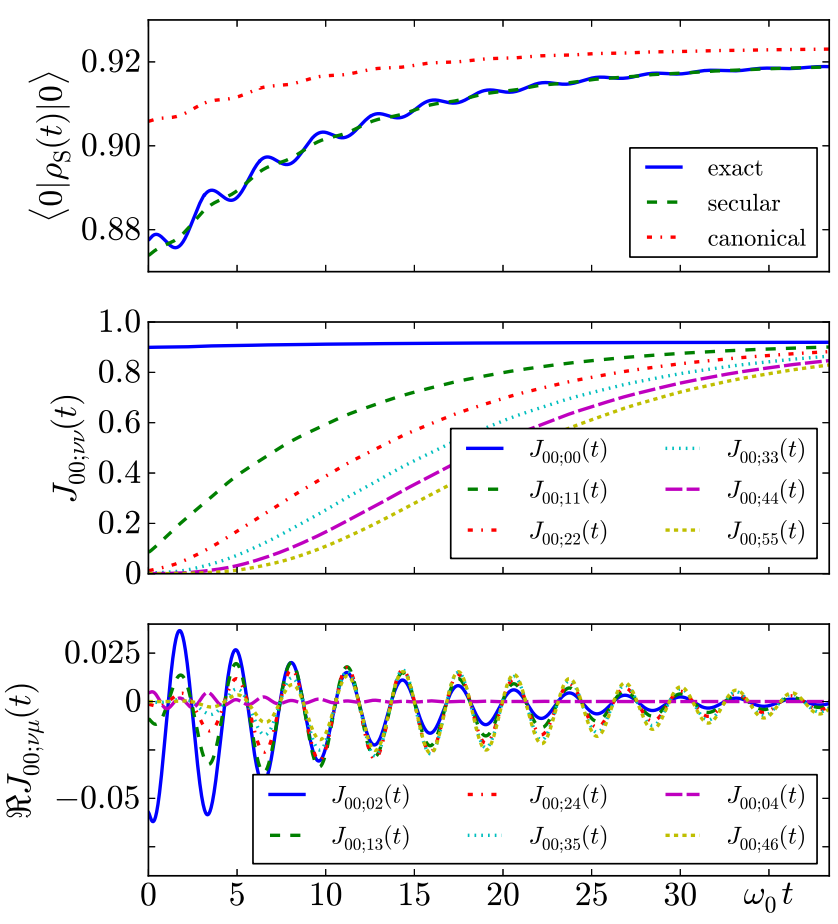

FIG. 7. (Color online). Upper panel: exact (continuous blue curve), secular-approximated (dashed green curve) time evolution of $\left\langle 0\left|\hat{\rho}_{\mathrm{S}}\right| 0\right\rangle$. The evolution using the canonical distribution as the initial state is depicted by the dot-dashed red curve. Lower panels: time evolution $J_{00 ; \nu \mu}$ for some values of $\nu$ and $\mu$. Parameters for coupling to TB as in Fig. 6, with $\hbar \omega_{0} / k_{\mathrm{B}} T_{\mathrm{BB}}=0.3884$, and $\Omega_{\mathrm{BB}}=8.3 \times 10^{5} \omega_{0}$.

In summary, irradiating with blackbody radiation cannot generate a coherent dynamical result, since the blackbody radiation is correctly represented as a thermal bath [see Eq. (12)]. Any time evolution observed here can be understood as the ultrafast transients as the system thermalizes, mediated by the driving initial-conditiondependent-transient-term. This term is present given the radiation field case, but is absent when excitation results from a second thermal bath coupled through the position of the bath modes.

\section{DISCUSSION AND CONCLUDING REMARKS}

We have considered the natural process where a system, originally coupled to a thermal bath, is subsequently perturbed by either another thermal bath or by blackbody
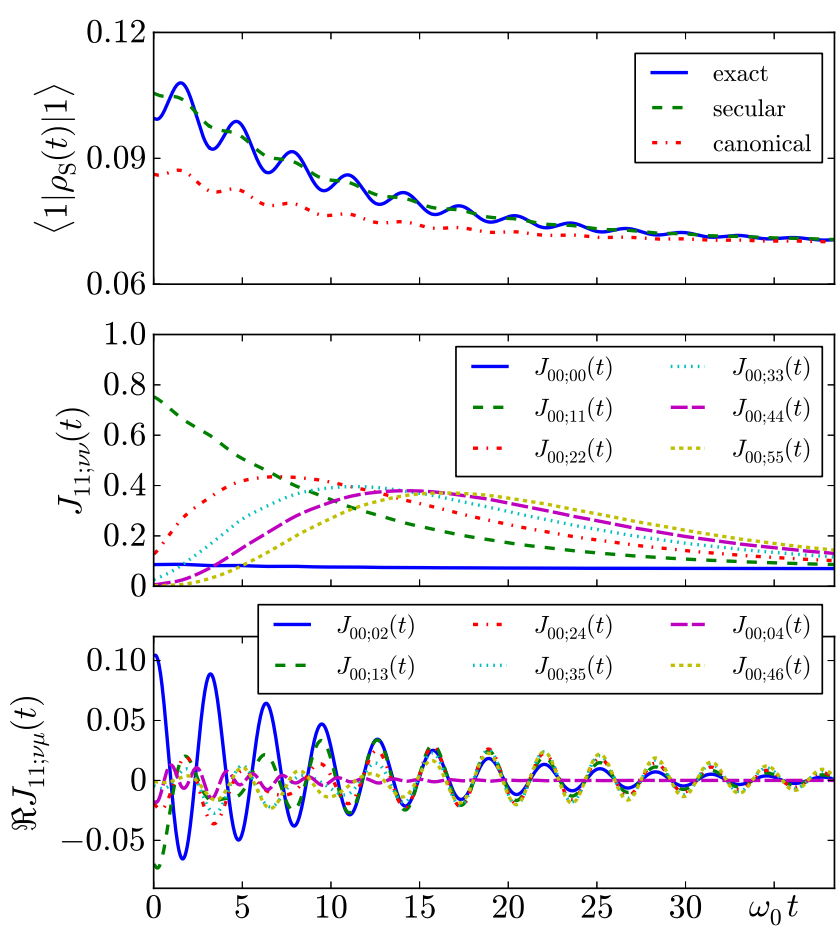

FIG. 8. (Color online). Time evolution of $\left\langle 1\left|\hat{\rho}_{\mathrm{S}}\right| 1\right\rangle$. Description and parameters as in Fig. 7

radiation. We have shown that the first step of thermalization generates off-diagonal stationary coherences which, in principle, could affect the dynamics of the second perturbative step. In particular, these off-diagonal stationary coherences could affect the populations of states during the second step. This process is enhanced in the low temperature regime, and plays a fundamental role in explaining the origin of one photon phase control in molecular systems [42, 43]. However, although our formal approach clearly identifies the stationary off-diagonal system matrix elements as participants in the subsequent time evolution of the populations of a generic open quantum system, when these off-diagonal terms are generated in practice, i.e. in natural environments or by blackbody irradiation, their contribution is negligible. This leads us to the conclusion that under natural conditions, the off-diagonal elements generated by thermal baths do not play any relevant role in the dynamics and that incoherent excitation of an open quantum system leads to dynamics free of coherent time evolution after an initially short transient time interval. Incoherent dynamics do occur, however, such as heat flux between the two baths via the system S [44].

A final note is in order. Our description of TB resembles, e.g., the role of a solvent. However, if the bath is part of the same macromolecule, then the spectral density $J_{\mathrm{TB}}(\omega)$ could be highly structured and some new features may be expected, e.g., increasing the stationary coherences even with weak decay rates. In those cases, the role 
of stationary coherences has to be explicitly calculated for each system at hand.

\section{ACKNOWLEDGMENTS}

LAP acknowledges discussions with Prof. Yehiam Prior and Dr. Timur V. Tscherbul with pleasure. This work was supported by the US Air Force Office of Scientific Research under contract number FA9550-10-1-0260, by Comité para el Desarrollo de la Investigación -CODI- of Universidad de AntioquiaColombia under contract number E01651 and under the Estrategia de Sostenibilidad 2013-2014, and by the Departamento Administrativo de Ciencia, Tecnología e Innovación -COLCIENCIAS- of Colombia under the project number 111556934912 .
[1] G. S. Engel, T. R. Calhoun, E. L. Read, T.-K. Ahn, T. Mančal, Y.-C. Cheng, R. E. Blankenship, and G. R. Fleming, Nature 446, 782 (2007).

[2] V. I. Prokhorenko, A. M. Nagy, S. A. Waschuk, L. S. Brown, R. R. Birge, and R. J. Miller, Science 313, 1257 (2006).

[3] X.-P. Jiang and P. Brumer, J. Chem. Phys. 94, 5833 (1991).

[4] H. Hoki and P. Brumer, Chem. Phys. Lett. 468, 27 (2009).

[5] H. Hoki and P. Brumer, Procedia Chem. 3, 122 (2011).

[6] T. Mančal and L. Valkunas, New J. Phys. 12, 065044 (2010).

[7] P. Brumer and M. Shapiro, Proc. Natl. Acad. Sci. U.S.A. 109, 19575 (2012).

[8] A. C. Han, M. Shapiro, and P. Brumer, arxiv.1211.5794 (2012).

[9] B. Xiao, B. Coste, J. Mathur, and A. Patapoutian, Nat Chem Biol 7, 351 (2011).

[10] E. Collini, C. Y. Wong, K. E. Wilk, P. M. G. Curmi, P. Brumer, and G. D. Scholes, Nature 463, 644 (2010).

[11] H. Hwang and P. J. Rossky, J. Chem. Phys. 120, 11380 (2004).

[12] H. Hwang and P. J. Rossky, J. Phys. Chem. B 108, 6723 (2004).

[13] I. Franco, M. Shapiro, and P. Brumer, J. Chem. Phys. 128, 244905 (2008).

[14] I. Franco and B. P., J. Chem. Phys. 136, 144501 (2012).

[15] M. Schlosshauer, Decoherence and the Quantum-ToClassical Transition (Springer-Verlag, Berlin, 2007).

[16] L. A. Pachón and P. Brumer, J. Phys. Chem. Lett. 2, 2728 (2011), arXiv:1203.3978.

[17] L. A. Pachón and P. Brumer, Phys. Chem. Chem. Phys. 14, 10094 (2012), arXiv:1203.3978.

[18] N. Bloembergen, Nonlinear Optics (Bejamin, New York, 1965).

[19] Y. Prior, A. R. Bogdan, M. Dagenais, and N. Bloembergen, Phys. Rev. Lett. 46, 111 (1981); N. Bloemberger, in Coherence, Cooperation and Fluctuations, Cambridge Studies in Modern Optics, Vol. 5, edited by F. Haake, L. M. Narducci, and D. F. Walls (Cambridge University Press, Cambridge, 1986) pp. 171-184; L. Rothberg, in Progress in Optics, Progress in Optics, Vol. XXIV, edited by E. Wolf (North-Holland, Amsterdam, 1987) pp. 41-101.

[20] N. Singh and P. Brumer, Faraday Disc. 153, 41 (2011).

[21] A. O. Caldeira and A. L. Leggett, Physica A 121, 587
(1983).

[22] H. Grabert, P. Schramm, and G.-L. Ingold, Phys. Rep. 168, 115 (1988).

[23] G. W. Ford, J. T. Lewis, and R. O'Connell, Phys. Rev. Lett. 55, 2273 (1985); Phys. Rev. A 36, 1466 (1987); G. W. Ford, J. T. Lewis, and R. F. O'Connell, Phys. Rev. A 37, 4419 (1988).

[24] F. Galve, L. A. Pachón, and D. Zueco, Phys. Rev. Lett. 105, 180501 (2010), arxiv:1002.1923.

[25] R. P. Feynman and F. L. Vernon, Annals of Physics 24, 118 (1963).

[26] U. Weiss, Quantum Dissipative Systems, 3rd ed. (World Scientific, Singapore, 2008).

[27] L. A. Pachón and P. Brumer, (2012), arxiv:1207.3104.

[28] M. Mohseni, P. Rebentrost, S. Lloyd, and A. AspuruGuzik, J. Chem. Phys. 129, 174106 (2008).

[29] M. B. Plenio and S. F. Huelga, New J. Phys. 10, 113019 (2008).

[30] S. Mukamel, Principles of Nonlinear Optical Spectroscopy (Oxford University Press, 1999).

[31] S. S. Goldstein, J. L. Lebowitz, R. Tumulka, and N. Zanghì, Phys. Rev. Lett. 96, 050403 (2006); S. Popescu, A. J. Short, and A. Winter, Nature Phys. 2, 754 (2006).

[32] L. A. Pachón and J. F. Triana, In preparation (2013).

[33] H. Grabert, U. Weiss, and P. Talkner, Z. Phys. B 55, 87 (1984).

[34] P. Hänggi and G.-L. Ingold, Chaos 15, 026105 (2005).

[35] Y.-C. Cheng and G. R. Fleming, Ann. Rev. Phys. Chem. 60, 241 (2009).

[36] P. Schramm and H. Grabert, Phy. Rev. A 34, 4515 (1986).

[37] L. A. Pachón, G. L. Ingold, and T. Dittrich, Chem. Phys. 375, 209 (2010), arXiv:1005.3839.

[38] P. M. V. B. Barone and A. O. Caldeira, Phys. Rev. A 43, 57 (1991).

[39] C. L. Mehta and E. Wolf, Phys. Rev. 134, A1143 (1964); Phys. Rev. 134, A1149 (1964); Phys. Rev. 161, 1328 (1967).

[40] G. W. Ford and R. F. O'Connell, Phys. Lett. A 45, 217 (1991); R. F. O'Connell, Phys. Lett. A 313, 491 (2003).

[41] G. W. Ford and R. F. O'Connell, Phys. Rev. A 57, 3112 (1998).

[42] L. A. Pachón, L. Yu, and P. Brumer, Faraday Disc. (2013), 10.1039/C3FD20144A, arxiv:1212.6416.

[43] L. A. Pachón and P. Brumer, In preparation (2013).

[44] L. A. Pachón and P. Brumer, In preparation (2013). 